\title{
Lo fugaz del instante jugable en los mundos virtuales: Espacialidad y temporalidad en SuperHOT y SuperHOT VR
}

Diego Villabrille Seca | al375496@uji.es, Marta Martín Nuñez | mnunez@uji.es

Universidat Jaume I, Castellón (España).

\author{
Resumen \\ Palabras clave \\ videojuegos; espacio; tiempo; realidad virtual; \\ SuperHOT; fugacidad \\ Sumario \\ 1. Introducción y planteamiento \\ metodológico. \\ 2. El gameworld. El diseño espacial como \\ tablero de juego. \\ 3. La construcción de la experiencia temporal \\ en el videojuego. \\ 4. Resultados del caso de estudio: SuperHOT. \\ 4.1. Time only moves if you do. La \\ construcción de la fugacidad a través de la \\ experiencia espacio-temporal. \\ 4.2. Suspense y bucle: jugar un shooter como \\ una partida de ajedrez. \\ 4.3. La agencia del jugador y la falacia del \\ control. \\ 4.4. La reconfiguración espaciotemporal en \\ SuperHOT VR. \\ 5 . Discusión y conclusiones. \\ 6. Bibliografía. \\ 7. Ludografía y filmografía.
}

La construcción de un tiempo y un espacio diegéticos es esencial para el relato de cualquier medio narrativo tradicional El videojuego —especialmente cuando despliega un desarrollo narrativo, bien a través de narrativas emergentes o estructuradastambién construye una temporalidad y una espacialidad propias que generan mundos ludoficcionales (Planells, 2015) que, a diferencia de los medios tradicionales, deben funcionar con la propia jugabilidad del sistema — esto es, el conjunto de mecánicas y reglas que permite el desarrollo lúdico-. El objetivo de este artículo es analizar el funcionamiento del tiempo y el espacio y los recursos que se utilizan para la construcción del relato, atendiendo a la forma en la que se relacionan con la jugabilidad del sistema. Para ello, nos centraremos en el caso de estudio de los videojuegos SuperHOT (SUPERHOT Team, 2016) y SuperHOT VR (SUPERHOT Team, 2018). (Fernández-Vara, 2015, Navarro-Remesal, 2016) y de análisis narrativo del videojuego (Planells, 2015) que complementaremos con conceptos de la narrativa tradicional (Gómez-Tarín, 2011). El análisis ofrecerá resultados sobre el grado de interrelación entre tiempo, espacio y mecánicas en la construcción de los relatos videolúdicos, así como los modos en los que los dispositivos de realidad virtual resignifican las nociones espaciotemporales.

\section{Cómo citar este texto:}

Diego Villabrille Seca y Marta Martín Nuñez (2020): Lo fugaz del instante jugable en los mundos virtuales. Espacialidad y temporalidad en SuperHOT y SuperHOT VR, en Miguel Hernández Communication Journal, Vol. 11 (2), pp. 221 a 238. Universidad Miguel Hernández, UMH (Elche-Alicante). DOI: http:// dx.doi. org/10.21134/mhcj.v11i0.340 


\section{The briefness of the playable instant in the virtual worlds: Spatiality and temporality in SuperHOT and SuperHOT VR}

Diego Villabrille Seca | al375496@uji.es,

Marta Martín Nuñez | mnunez@uji.es

Universidat Jaume I, Castellón (España).

\footnotetext{
Abstract

Keywords

"Video games"; "Space"; "Time"; "Virtual reality";

"SuperHOT"; "Briefness".

Summary

1. Introduction and methodological approach.

2. Gameworld. The design of space as a game board. 3.

Building time experience in video games.

4. Results of the case study: SuperHOT.

4.1. Time only moves if you do. The construction of

briefness through time and space experience.

4.2. Suspense and loop: playing a shooter as a chess game.

4.3. The player's agency and the fallacy of control.

4.4. The time-space reconfiguration in SuperHOT VR.

5. Discussion and conclusions.

6. Bibliography.

7. Ludography and filmography.
}

Constructing a diegetic time and space is essential for the storytelling of any traditional narrative medium. Video games —especially when deploying a narrative development, either through emerging narratives or structured ones-, also build a temporality and a spatiality of their own that develop ludofictional worlds (Planells, 2015) which, unlike traditional media, should work with the system's gameplay — this is, the logical set of mechanics and rules that allow for the development of play-. The aim of this paper is to analyse how time and space work and the elements used to build the storytelling, dealing with the way in which they are related to the gameplay of the system. In order to do this, we will focus on the video games SuperHOT (SUPERHOT Team, 2016) and SuperHOT VR (SUPERHOT Team, 2018) as case study. We will follow a methodology for video game analysis (Fernández-Vara, 2015, Navarro-Remesal, 2016) and video game narrative analysis (Planells, 2015) both of which we will complement with concepts about traditional narrative (GómezTarín, 2011). The resulting analysis will offer results about the degree of interconnectedness between time, space and game mechanics in the construction of video game narratives, as well as the ways in which virtual reality devices resignify the space-time notions.

\section{How to cite this text:}

Diego Villabrille Seca y Marta Martín Nuñez (2020): The briefness of the playable instant in the virtual worlds. Spatiality and temporality in SuperHOT and SuperHOT VR, in Miguel Hernández Communication Journal, Vol. 11 (2), pp. 221 a 238. Universidad Miguel Hernández, UMH (Elche-Alicante). DOI: http:// dx.doi. org/10.21134/mhcj.v11i0.340 


\section{Introducción y planteamiento metodológico}

Pensar en la representación de la fugacidad es pensar en aquellos medios que, como la fotografía, detienen el tiempo: "Temporalmente (...) la imagen-acto fotográfico interrumpe, detiene, fija, inmoviliza, separa, despega la duración captando solo un instante" (Dubois, 2015: 141). Ese instante fotográfico fija así un acontecimiento — tan fugaz como el tiempo que fluye- y, como una máquina del tiempo, nos permite volver a él a través de la representación. El videojuego, en cambio, se aleja de este principio: por lo general, huye de lo fugaz; más bien, le interesa la duración — promete horas de jugabilidad - y, salvo que tenga un alto valor de rejugabilidad - porque proporcione experiencias diferentes en cada partida_, pocas veces se retorna a él como se retorna a una fotografía en busca del recuerdo de un instante pasado. Porque el videojuego privilegia el presente, cuyo instante es diferente cada vez. Por eso, paradójicamente, aunque no invite al retorno, el videojuego invita a ser pensado desde la repetición, como un sistema en el que los jugadores, como si fueran sísifos, se enfrentan en bucle a unos obstáculos pensados para que no logren llegar a la cima de la montaña sin caer de nuevo abajo. No en vano, el académico Jesper Juul, habla del videojuego como el arte del fracaso (2013), en el que el placer proviene, precisamente, de demostrar una maestría sobre él (Hanson, 2018: 110). Sin embargo, la complejidad mecánica y ficcional de algunos juegos que experimentan con la alteración de los patrones espacio-temporales en las capas lúdicas y de ficción nos ha llevado a preguntarnos por el concepto de la fugacidad en el videojuego. Y no (solo) desde la detención del tiempo sino también desde la experiencia con él y las relaciones espaciales. El videojuego, como espacio de juego y de ficción, construye una temporalidad y una espacialidad que están interrelacionadas de forma intrínseca, generando un mundo de carácter ludoficcional (Planells, 2015). Aunque esta temporalidad y espacialidad normalmente se hacen especialmente patentes en el despliegue narrativo - ya sea a través de narrativas emergentes o estructuradas-, no pertenecen en exclusiva a este ámbito, y se ven inscritas en la propia jugabilidad del medio, construyéndose como parte esencial del sistema de reglas y mecánicas que permite el desarrollo del medio videolúdico.

El objetivo de esta investigación, por tanto, es analizar el funcionamiento interdependiente de estos sistemas de espacio y tiempo y cómo se relacionan con la construcción de la experiencia jugable a través de la jugabilidad y la narrativa. Para ello, tomaremos como caso de estudio SuperHOT (SUPERHOT Team, 2016) y su secuela adaptada a la realidad virtual, SuperHOT VR (SUPERHOT Team, 2018), como ejemplo paradigmático del grado de interrelación existente entre el tiempo y el espacio en el medio videolúdico. La elección de SuperHOT como caso de estudio se justifica porque, desde el nivel más esencial, que se encuentra en la capa legislativa del videojuego y que define las mecánicas y las reglas, a la capa más "accidental" (Monchán, 2014: 81), que es la capa de la narrativa estructurada, el juego explicita este aspecto de la naturaleza del medio videolúdico. Como indica Hanson (2018: 1), se trata de un juego construido completamente alrededor de la mecánica de la temporalidad distorsionada vinculada directamente al movimiento y la agencia del jugador. Por otra parte, la adaptación del videojuego a los dispositivos de realidad virtual, pasando de una representación bidimensional en pantalla, a un espacio inmersivo tridimensional, requiere de una nueva versión del videojuego que modifica y resignifica la construcción de la temporalidad y la espacialidad.

Para el análisis de ambos videojuegos, emplearemos herramientas metodológicas propias de los Game Studies basadas en el enfoque textual, que tiene como finalidad desvelar los mecanismos de construcción de la significación a partir de la interacción del jugador y de los sistemas y 
representación del propio videojuego. Para ello, tomaremos como referencia el modelo de Fernández-Vara (2015), que se aproxima al videojuego desde el análisis del contexto, los aspectos esenciales del juego y los elementos formales, y lo pondremos a dialogar con los postulados sobre el concepto de libertad dirigida que desarrolla Navarro-Remesal (2016), que define como el margen de acción que el sistema jugable permite al jugador a través de la comunicación con el sistema y la aproximación a los mundos ludoficcionales abordada por Planells (2015). Esta mirada desde los Game Studies la complementaremos también con conceptos de la narrativa audiovisual tradicional (Gómez-Tarín, 2011). El análisis de estos aspectos de ambos videojuegos —que entendemos y analizamos realmente como dos versiones de un mismo producto audiovisual — nos permitirá obtener resultados sobre el grado de interrelación entre el tiempo y el espacio en el medio videolúdico. Y, con ello, una comprensión de cómo estos se interrelacionan con las mecánicas para la construcción de las experiencias jugables.

\section{El gameworld. El diseño espacial como tablero de juego}

El diseño del espacio es parte de la naturaleza esencial del medio videolúdico: los videojuegos, por norma general, necesitan generar un mundo de ficción de algún tipo con el que el jugador pueda interactuar (Newman, 2004, en Gazzard, 2011). Tal y como indica Planells, los mundos ludoficcionales reciben la herencia de los mundos de ficción propios de la literatura y el cine en cuanto a su contenido, pero resultan distintos en cuanto a su capacidad proactiva y transformativa en relación con el usuario. Así, estos mundos tendrán una semántica intensional propia que les habilita para contener distintos tipos de personajes, objetos, espacios y temporalidades, pero deben asociarse, necesariamente, con la influencia ludológica de las reglas de juego (Planells, 2015: 98). De este modo, el diseño del gameworld se puede concebir como el tablero de juego necesario que plantea el marco ficcional, pero que está sometido a las reglas del juego. Además, si existe un desarrollo narrativo, este marco ficcional será también donde tengan lugar las acciones y eventos que, además, nos aporta información valiosa sobre el tema, lo que allí ocurre, y las convenciones y tramas que puede evocar al jugador (Fernández-Vara, 2015: 104).

La importancia del diseño espacial para la significación del videojuego ha llevado a autores como Jenkins (2004) a afirmar que la profundidad de un videojuego tiene más que ver con diseñar mundos y esculpir espacios que con los eventos narrativos o las motivaciones de los personajes. $\mathrm{Y}$, efectivamente, esto tiene que ver con el diseño de la experiencia jugable. A diferencia de otros medios, como el cinematográfico, donde la cinta avanza independientemente de la actitud o atención del espectador, un videojuego, por lo general, necesita de la acción del jugador para avanzar. El jugador debe ejecutar las mecánicas que le permite el juego —andar, correr, hablar con otros personajes, buscar elementos, resolver puzles, luchar, etc. - para avanzar en su experiencia y hacer avanzar también el desarrollo del juego. No es de extrañar, por ello, que muchos juegos — desde obras independientes como Gone Home (Fullbright, 2013) hasta superproducciones como Death Stranding (Kojima Productions, 2019) - basen su experiencia de juego en el concepto de exploración del espacio y en la profundización en el mundo que se nos propone. Algunos académicos como Ruiz Collantes (2008: 19) nos proponen la idea del videojuego como "vivencia narrativa" para describir la experiencia cognitiva, emocional y sensorial que implica el proceso de juego y admite que, a través de la narración performática, el jugador explora el entorno y, con sus elecciones, impulsa y genera su propia vivencia narrativa, por lo que, de nuevo, la idea de exploración y recorrido se conforman como el núcleo del diseño de la experiencia jugable.

A través del diseño representacional, el videojuego propone al jugador un espacio virtual que 
tiene como única limitación física real los límites de la pantalla. Pero el mundo virtual es un mundo que el jugador pasa a habitar a través de la inmersión, cuyos límites pueden estar más allá de la pantalla (Cabañes, 2013). Es, al fin y al cabo, el jugador quien interviene la imagen para comprenderla como un espacio y hacerlo suyo, necesariamente condicionado por la mirada de quien interpreta la imagen, y genera un mapa del mundo, en toda su literalidad. No tiene sentido entender el espacio virtual como un lugar con significado fuera de la experiencia jugable. Como apunta Nitsche:

Virtual space is a product of human knowledge and depends on logic, software, and hardware development. [...]. It is a world defined by a universe of coordinates. That is why at its core and at the moment of its creation, it is spatial but not a place. In order for these data visualizations to become meaningful, they have to be engaged by the player. Through the active work of the player, through comprehension and interaction, the masses of polygons can transform into places. (2008: 191) ${ }^{1}$.

Estos lugares, que adquieren forma y sentido en la medida en la que el jugador los ejecuta, se conforman como la base sobre la que se construirá también la percepción del tiempo, dando lugar al gameworld o mundo jugable. Es este mundo el que vertebrará posteriormente el proceso lúdico y el espacio principal donde tiene lugar el proceso de comunicación bidireccional entre el sistema y el jugador. Aquí es donde el jugador pueda relacionarse con el sistema a través de las mecánicas, poniendo a dialogar sus deseos con las reglas propuestas y se enfrente así a las obligaciones, prohibiciones, penalizaciones o premios del sistema por sus acciones (NavarroRemesal, 2015). No puede existir videojuego sin espacio ludoficcional, porque, si bien es un lugar que solo existe en el contexto del propio proceso lúdico, su aparición es intrínseca al propio hecho del juego.

\section{La construcción de la experiencia temporal en el videojuego}

La temporalidad es una característica, de nuevo, esencial en la experiencia de juego, vinculada a la experiencia de la tragedia (Vargas-Iglesias, 2019). En palabras de Bushnell, "while engaging the player as spectator, actor and author in that experience, videogames thus both draw on and revert back to the essential tensions inherent in the tragic experience of time"2 (2016: 80). Los videojuegos, además, recogen la experiencia narratológica escrita sobre lo que Genette (1980: 34) llamó el pseudo-tiempo para referirse al tiempo ficcional que se refiere al tiempo real/ verdadero — true - necesario para cruzar o atravesar el espacio — un libro, que también se puede aplicar a un videojuego- - Pero más allá del pseudo-tiempo, Eskelinen suma a la narrotología cybertextual los conceptos de tiempo del sistema —el grado variable de la permanencia del texto que está accesible para el usuario-, y el tiempo de lectura —el potencial de controlar la accesibilidad temporal del usuario_- Por encima de esta capa, suma una tercera capa, la del tiempo real/ de la realidad — como real_ y se refiere a la comunicación textual en tiempo real entre usuarios dentro del texto como una parte fundamental de su consumo y construcción (2012: 134-137).

${ }^{1} \mathrm{El}$ espacio virtual es un producto del conocimiento humano y depende del desarrollo de la lógica, el software y el hardware. [...]. Es un mundo definido por un universo de coordenadas. Por eso en su núcleo y en el momento de su creación, es espacial pero no un lugar. Para que estas visualizaciones de datos sean significativas, tienen que ser realizadas por el jugador. A través del trabajo activo del jugador, a través de la comprensión y la interacción, las masas de polígonos pueden transformarse en lugares.

${ }^{2} \mathrm{Al}$ mismo tiempo que involucra al jugador como espectador, actor y autor en esa experiencia, los videojuegos se basan y, al mismo tiempo, vuelven a las tensiones esenciales inherentes a la trágica experiencia del tiempo. 
Esta conceptualización temporal nos muestra que es necesaria la existencia del espacio para la percepción de esta temporalidad, ya que es la continuidad del espacio la que actúa como factor relacional que permitirá la conexión entre espacio y movimiento que da lugar a la temporalidad en el videojuego, permitiendo que los jugadores no tengan problemas a la hora de convivir con los paradigmas temporales en los juegos (Sora, 2016). Esto es, el movimiento de los objetos en el espacio virtual percibido es lo que permite la creación de un sistema temporal a través de la proyección mental de este movimiento en el espacio percibido, sin necesidad de una presencia corpórea (Nitsche, 2007). Tomemos, por ejemplo, Tetris (The Tetris Company, 1984). Entendemos que existe una progresión temporal durante el gameplay únicamente debido a la existencia del movimiento de las piezas en la pantalla, que actúan como límite del espacio del mundo virtual. De pararse el movimiento de las piezas del juego no habría percepción alguna del tiempo y, en consecuencia, no habría jugabilidad posible. Si, por otra parte, las piezas comenzaran a moverse desde la parte inferior de la pantalla y a realizar el mismo movimiento que previamente habían realizado, pero a la inversa, el jugador entendería que sucede una suerte de reversión del eje temporal, precisamente por la existencia de continuidad en el espacio que permite entender el paradigma temporal que se presenta.

Así vemos cómo la temporalidad de un mundo ludoficcional está estrechamente vinculada al espacio donde se desarrolla, y es solamente a través de la combinación de ambos ejes cuando se abre la posibilidad de generar un sistema en el que el jugador pueda tener un cierto nivel de interacción y agencia con ese mundo. La configuración de ese espacio y ese tiempo son, junto con las mecánicas, en definitiva, la cristalización de las reglas del mundo. En un videojuego el jugador no necesariamente aprende directamente las reglas del juego, sino que aprende la forma de jugar (Lindley, 2005), es decir, la forma de habitar un mundo, o los patrones de movimientotiempo en un espacio.

Más allá de la idea de que el jugador actúa y ejecuta mecánicas en tiempo real y, por tanto, el tiempo en un videojuego está necesariamente conectado con el tiempo presente del jugador, debemos detenernos en dos aspectos fundamentales que añaden cierta complejidad a la temporalidad en el videojuego. Por una parte, a nivel mecánico, debemos atender el hecho de que muy pocos juegos se juegan en línea recta: la mayor parte de los juegos se juegan en círculos, a partir de bucles temporales que permiten repetir un nivel —o una acción — una y otra vez hasta completarlo en una repetición iterativa ${ }^{3}$ en consonancia con la lógica algorítmica del videojuego. El horizonte de ciclos infinitos de muerte-resurrección asumidos en la cultura videolúdica nos lleva incluso a entender el videojuego como un discurso del fracaso, tal y como lo ha expresado Jesper Juul (2013), en el que el videojuego propone un entorno para el fracaso que permite al jugador experimentar con él y con la necesaria mejora de habilidades para superarlo, convirtiéndose así en el núcleo del goce del videojuego. Precisamente, la serie de juegos conceptuales que propone el diseñador Pippin Barr en Lets Play: Ancient Greek Punishment (Pippin Barr, 2011) en los que es imposible ganar, buscan transgredir esta idea de éxito. Más allá del bucle propio del videojuego, son muchos los juegos en cuyas mecánicas principales interviene el aspecto temporal, ya que no solo importa la acción realizada, sino cuándo es realizada — como vemos en la mayor parte de videojuegos de plataformas y que en Portal (Valve, 2007) adquiere gran importancia-, o incluso mecánicas diseñadas para resolver puzles temporales a partir de la manipulación del tiempo, como vemos en juegos como Braid (Number None, 2008).

${ }^{3}$ Entendemos iterativa en el sentido algorítmico, es decir, no como una simple repetición, sino como un proceso que se aproxima a un resultado mediante la aplicación repetida de una serie de instrucciones (jugar el nivel, en este caso) que produce unas salidas que se utilizan para modificar las entradas en cada una de las iteraciones (intentos). 
Por otra parte, aunque el videojuego siempre se juega en presente, como hemos apuntado antes, en los videojuegos narrativos es común que el relato, en la estela de las narrativas postclásicas como los mind-game films (Elsaesser, 2009, 2013), nos plantee alteraciones temporales del desarrollo cronológico de la narración. Juegos como Beyond: Two Souls (Quantic Dream, 2013) precisamente nos plantean la posibilidad de jugar la vida de Jodie y Aiden a través de saltos entre distintos momentos de su historia, trasladándonos continuamente entre episodios de su infancia, su vida como agente de la CIA o su vida alejada de todo lo conocido.

Sin embargo, las temporalidades más complejas ocurren cuando la capa mecánica y la capa narrativa se conjugan para posibilitar mecánicas temporales que tienen una significación directa sobre la narrativa. Por ejemplo, en Life is Strange (Dontnod Entertainment, 2015) observamos cómo la mecánica principal — la capacidad del avatar del jugador de retroceder en el tiempo de la diégesis y así tomar decisiones diferentes - está directamente relacionada con el sentimiento de culpa que asola a Max, la protagonista, incapaz de salvar a su pueblo y a su mejor amiga al mismo tiempo (Martín Núñez, García Catalán y Rodríguez Serrano, 2016). La temporalidad, por tanto, se convierte en un elemento del mundo ludoficcional que puede llegar a albergar una gran complejidad al actuar en las diferentes capas de significación del videojuego.

\section{Resultados del caso de estudio: SuperHOT}

\subsection{Time only moves if you do. La construcción de la fugacidad a través de la experiencia espacio-temporal}

La relación entre el tiempo y el espacio como elementos del mundo del juego queda patente desde el principio en SuperHOT. Al comenzar la simulación, la voz del narrador queda sobreimpresionada en grandes letras sobre la pantalla del juego enunciando la premisa principal sobre la que girará la jugabilidad: "time only moves if you do" (el tiempo solo se mueve si tú lo haces). Desde el momento que comenzamos el juego, se nos presenta una interrelación total entre el tiempo y el espacio que lleva al extremo la idea de avance en el videojuego. Si normalmente necesitamos de un jugador activo que avance en el espacio para avanzar en el juego, aquí, esta idea se potencia mostrando cómo el tiempo virtual es, en definitiva, movimiento. Lo que se está indicando en realidad es que el resto de las entidades del escenario solo se moverán cuando el jugador lo haga, construyendo así un tiempo en el que se explicita que este solo existe en tanto que movimiento en un espacio virtual.

Sin embargo, tras esta premisa — falaz, como veremos más adelante- lo que se esconde en realidad es un juego de temporalidades, donde conviven dos velocidades distintas: la del juego, que es tan lenta que parece que no avanza, casi inmóvil; y la del personaje-jugador que, al moverse a una velocidad normal, parece que sea extremadamente veloz —al menos, en comparación con la velocidad del mundo de juego- . De este modo, la sensación que tenemos al ejecutar mecánicas a una velocidad natural en un mundo hiperralentizado es que caminamos, disparamos o atacamos a una velocidad extrema, generando la falsa impresión de que nuestros reflejos son rapidísimos. La idea de fugacidad, pues, se construye contraponiendo estos dos tempos, haciendo experimentar el tempo natural como un tiempo veloz por comparación en un mundo en el que parece que nada se mueve.

${ }^{4}$ Entendemos Aunque fue la película de Matrix la que popularizó el bullet time, ya había sido antes utilizado antes por Michael Gondry en un videoclip de los Rolling Stones. 
La ilusión de fugacidad que experimentamos al jugar el juego está basada en el efecto bullet time que popularizó la película Matrix (The Matrix, Hermanas Wachowski, 1999) ${ }^{4}$ —referencia nada arbitraria, como analizaremos más adelante-, como vemos en la figura 1. La ralentización extrema de la acción permite visualizar acontecimientos muy rápidos, normalmente invisibles para el ojo humano, como la trayectoria de una bala. Al mismo tiempo que se ralentiza la acción, se mueve la cámara — en el juego, en manos del jugador — desplazando el ángulo de visión para crear efectos dramáticos. SuperHOT utiliza este efecto como mecánica, permitiendo que en este juego de tempos el jugador pueda transitar ambas velocidades e incluso cortar la bala por la mitad con una catana.
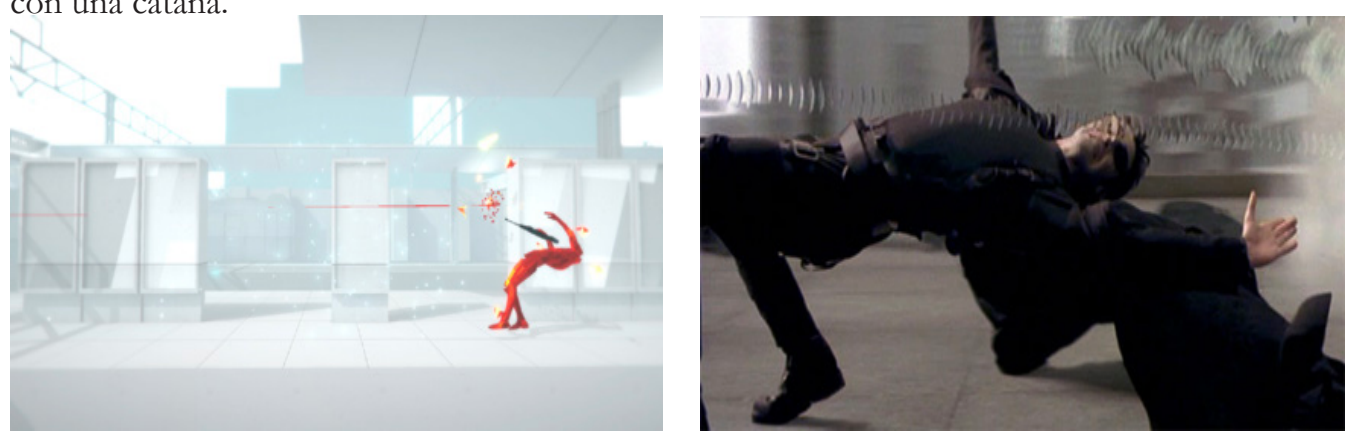

Fig. 1. Fotograma de SuperHOT (2016), SUPERHOT Team C) y de Matrix (Hermanas Wachowski, 1999), Warner Bros. Entertainment Inc. (C) en las que se aprecian las similitudes visuales en la construcción del efecto visual bullet time.

Otro elemento visual que potencia la idea de fugacidad del mundo ludoficcional es la explosión de los enemigos cuando el jugador les dispara o ataca. Las figuras rojas, de estilo low-poly, parecen explosionar en mil pedazos como si fuesen jarrones de cristal cuyos añicos permanecen suspendidos en el aire durante unos segundos cuando reciben un impacto. Este efecto que, como la trayectoria de la bala, es habitualmente tan rápido que pasa desapercibido para el ojo humano, aquí se alarga para el deleite visual —y mecánico, al fin y al cabo—. Sin duda, este efecto ha sido explotado antes también por la fotografía, cuya naturaleza como medio invita a congelar instantes para la memoria. Obras como Rapid Bloom de Martin Klimas giran precisamente en torno al registro de lo que es invisible para el ojo. El artista congela con nitrógeno líquido flores que después hace explosionar para captar la fragilidad de la belleza que escapa, y cuyos pedazos suspendidos en el aire establecen un paralelismo inquietante con los polígonos de los enemigos en SuperHOT.
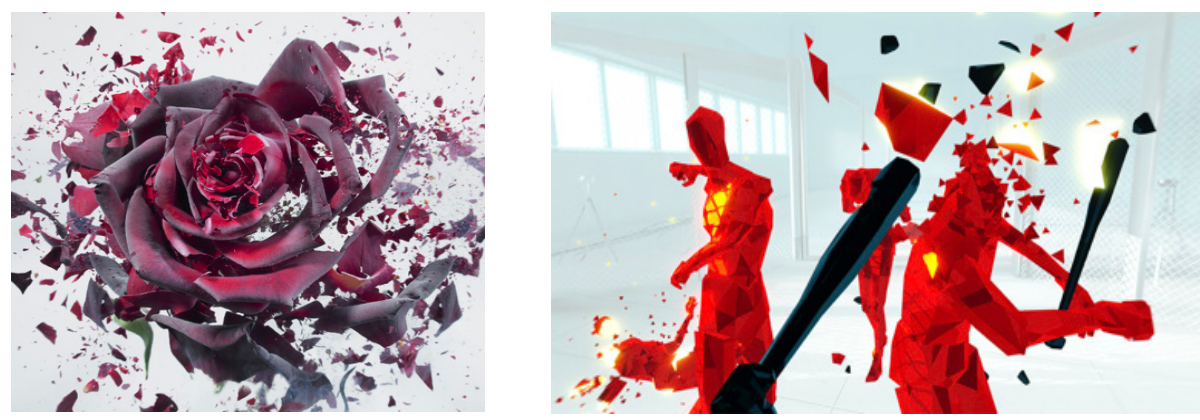

Fig. 2. Fotograma de SuperHOT (2016), SUPERHOT Team (C) y fotografía de Martin Klimas, Rapid Bloom (2013). 
SuperHOT, al poner en manos del jugador el vector espacio-temporal, le confiere una agencia que lo carga de un aparente control sobre el mundo de juego. Esta agencia del jugador sobre el conjunto de elementos del entorno a través del vector espacio-temporal es una muestra del carácter performativo del videojuego, que sitúa al jugador en un paradigma de interpretación activa e intervención en el mundo de juego. Esto hace de cada momento un instante doble fugaz e irrepetible, por una parte, por la interpretación activa, y por otra, por la capacidad de actuación sobre el entorno.

\subsection{Suspense y bucle: jugar un shooter como una partida de ajedrez} SuperHOT se ha llegado a describir como un shooter por turnos, ya que el jugador debe pensar cada movimiento que hace, pues este hará avanzar el tiempo y, con él, los enemigos y la trayectoria de sus balas, como en una partida de ajedrez. Ralentizar el tiempo implica necesariamente pensar cada movimiento, uno detrás de otro, como si fuese una coreografía cuyo objetivo es acabar con todos los enemigos, que se aproximan a nosotros desde distintos ángulos y cuyas balas dibujan amenazadoras trayectorias hacia la cámara — la posición del jugador- Esta suspensión del tiempo entre movimientos desata el suspense, la espera - ese tiempo para pensar- entre movimientos, genera una expectación impaciente por la resolución de cada acción, cuyo origen ya no es el tempo del relato, como en un film, sino el tiempo que el propio jugador necesita para pensar. Si en un shooter normal, la acción frenética desborda al jugador, generando una sensación donde se suspende el pensamiento y se descarga adrenalina, SuperHOT invierte la mecánica: suspende el tiempo para suspender el movimiento, y así genera espacios de espera donde aumenta la tensión, cuya descarga se produce cada vez que se finaliza cada nivel.

Aquí es donde adquiere relevancia el concepto de bucle temporal que se desata en cada nivel. Siendo este un recurso temporal habitual en los videojuegos, en SuperHOT se ve claramente reforzado mediante el mensaje que incita a reiniciar rápidamente el nivel en el momento en el que el jugador recibe un disparo "press $\mathrm{R}$ to restart instantly" (pulsa $\mathrm{R}$ para reiniciar instantáneamente). Pero este bucle no hace sino que reforzar la fugacidad de cada momento de la experiencia temporal del juego, porque no invita al jugador a repetir las mismas acciones, sino, a repensar sus movimientos, convirtiéndose en un elemento vital en el proceso de aprendizaje. Esto hará que en cada nuevo intento el jugador no realice el proceso de interpretación y actuación sobre el gameworld de la misma forma, sino que lo reformule en función del conocimiento necesariamente adquirido con cada iteración.

Pero el bucle no acaba con el desempeño exitoso de cada nivel: con cada nivel superado este se manifiesta de nuevo, y el juego reproduce lo jugado, pero esta vez en tiempo real —entendiendo por tiempo real que el tiempo de la diégesis - mientras escuchamos y vemos sobreimpreso un mensaje que repite incesantemente el nombre del juego, como si de la profesión a una deidad se tratase-. En esta suerte de killcam invertida — pues nos muestra la victoria, que percibimos como a cámara rápida-, la velocidad de movimiento de las entidades en el espacio virtual pasa a mostrarse desvinculada de la velocidad de movimiento del jugador, que en la reproducción se mueve a una velocidad fija, de nuevo probando el hecho de que el tiempo percibido por el jugador no es más que la interpretación del movimiento de los objetos en un espacio virtual.

\subsection{La agencia del jugador y la falacia del control}

Es aquí donde cabe plantearse más en detalle la premisa espacio-temporal planteada por SuperHOT. Desde el inicio del juego, al jugador se le invita a pensar que controla el tiempo de la diégesis con 
sus movimientos — "time only moves if you do", indica el narrador mediante una sobreimpresión que cubre toda la pantalla- Pero esta regla de acción es directamente falsa. El tiempo sí avanza cuando el jugador no interactúa con el entorno. Si el jugador no hace uso de su agencia en el entorno y se limita a quedarse quieto, las demás entidades seguirán moviéndose, aunque muy lentamente.

El hecho de que el jugador carezca de control real sobre el tiempo del mundo virtual impulsa definitivamente la imposibilidad de la repetición del instante de juego, reforzando la naturaleza fugaz del medio videolúdico. Pero más allá de esto, esta mentira oculta la verdad más universal del videojuego como medio: los sistemas videolúdicos operan en una ilusión de libertad para con el jugador. La agencia del avatar del jugador sobre el entorno está limitada por los designios de la enunciación que, a través del sistema de reglas, da lugar al mundo ludoficcional —y su construcción espacio-temporal-y permite interactuar con él a través de mecánicas, o como lo ha enunciado Navarro-Remesal, la libertad está dirigida (2016). En SuperHOT el jugador no posee el control del tiempo, solo está bajo una ilusión de la tener tal capacidad. La ausencia de un menú de pausa en consonancia con los estándares del videojuego refuerza la imposibilidad de parar — atrapar - el tiempo de un juego en el que, en un principio, pensamos que el tiempo está precisamente bajo nuestro control como jugadores. Sin embargo, la fiabilidad en el narrador se va quebrando a medida que avanza el juego: a veces utilizará su voz para dar órdenes — disfrazadas de indicaciones de juego- - pero otras, directamente hará comentarios irónicos sobre la situación como "reunión aplazada" — al asesinar a todos los PNJ de una oficina_ o "elevator pitch" —al presentar una escena en un ascensor- En ocasiones puntuales rompe la cuarta pared y se burla directamente el jugador, recordándole que no está en control de la situación al encerrarlo en una habitación sin salida, darle órdenes y, al cumplirlas, felicitarle con: "buen perro". Quedar en manos de un narrador poco fiable, un rasgo de las narrativas postmodernas (Sorolla-Romero, 2018), es algo que ya se ha visto en otros juegos metadiscursivos como The Stanley Parable (Galactic Cafe, 2011), donde se reflexiona precisamente sobre la libertad de elección y los deseos del jugador durante la experiencia jugable.

En el caso que nos ocupa, lo cierto es que la parte jugable a la que nos hemos referido hasta ahora en SuperHOT es, en realidad, una simulación a la que accedemos a través de un sistema informático clásico tipo MS-DOS, en la que nos salta un chat de un supuesto amigo que nos invita a instalarnos un ejecutable del juego del que dice no haber visto nada parecido. En este sistema se desarrolla toda la narrativa estructurada del juego y, como si de una rima se tratase, gira esencialmente en torno a la limitación de la libertad del jugador y su control mediante un sistema informático. Así, el videojuego tiene una lectura metadiscursiva que refuerza la idea del juego como libertad limitada por el sistema de reglas y mecánicas. El paralelismo existente con la premisa de Matrix y los interfaces que emplea potencian también esta interpretación metadiscursiva del videojuego. No obstante, como hemos estudiado a propósito de otros juegos (Rodríguez Serrano, Martín-Núñez y Gil-Soldevila, 2017), es probable pensar que el interés en el juego no descansa principalmente en la actualización narrativa de un universo conocido - el usuario contra el sistema - sino en la novedad mecánica que el juego propone.

\subsection{La agencia del jugador y la falacia del control}

Es aquí donde cabe plantearse más en detalle la premisa espacio-temporal planteada por SuperHOT. Desde el inicio del juego, al jugador se le invita a pensar que controla el tiempo de la diégesis con sus movimientos - "time only moves if you do", indica el narrador mediante una sobreimpresión que cubre toda la pantalla_- Pero esta regla de acción es directamente falsa. El tiempo sí avanza cuando el jugador no interactúa con el entorno. Si el jugador no hace uso de su agencia en el entorno y se limita a quedarse quieto, las demás entidades seguirán moviéndose, 
aunque muy lentamente.

El hecho de que el jugador carezca de control real sobre el tiempo del mundo virtual impulsa definitivamente la imposibilidad de la repetición del instante de juego, reforzando la naturaleza fugaz del medio videolúdico. Pero más allá de esto, esta mentira oculta la verdad más universal del videojuego como medio: los sistemas videolúdicos operan en una ilusión de libertad para con el jugador. La agencia del avatar del jugador sobre el entorno está limitada por los designios de la enunciación que, a través del sistema de reglas, da lugar al mundo ludoficcional —y su construcción espacio-temporal - y permite interactuar con él a través de mecánicas, o como lo ha enunciado Navarro-Remesal, la libertad está dirigida (2016). En SuperHOT el jugador no posee el control del tiempo, solo está bajo una ilusión de la tener tal capacidad. La ausencia de un menú de pausa en consonancia con los estándares del videojuego refuerza la imposibilidad de parar - atrapar - el tiempo de un juego en el que, en un principio, pensamos que el tiempo está precisamente bajo nuestro control como jugadores. Sin embargo, la fiabilidad en el narrador se va quebrando a medida que avanza el juego: a veces utilizará su voz para dar órdenes — disfrazadas de indicaciones de juego-, pero otras, directamente hará comentarios irónicos sobre la situación como "reunión aplazada" — al asesinar a todos los PNJ de una oficina_ o "elevator pitch" —al presentar una escena en un ascensor- En ocasiones puntuales rompe la cuarta pared y se burla directamente el jugador, recordándole que no está en control de la situación al encerrarlo en una habitación sin salida, darle órdenes y, al cumplirlas, felicitarle con: "buen perro". Quedar en manos de un narrador poco fiable, un rasgo de las narrativas postmodernas (Sorolla-Romero, 2018), es algo que ya se ha visto en otros juegos metadiscursivos como The Stanley Parable (Galactic Cafe, 2011), donde se reflexiona precisamente sobre la libertad de elección y los deseos del jugador durante la experiencia jugable.

En el caso que nos ocupa, lo cierto es que la parte jugable a la que nos hemos referido hasta ahora en SuperHOT es, en realidad, una simulación a la que accedemos a través de un sistema informático clásico tipo MS-DOS, en la que nos salta un chat de un supuesto amigo que nos invita a instalarnos un ejecutable del juego del que dice no haber visto nada parecido. En este sistema se desarrolla toda la narrativa estructurada del juego y, como si de una rima se tratase, gira esencialmente en torno a la limitación de la libertad del jugador y su control mediante un sistema informático. Así, el videojuego tiene una lectura metadiscursiva que refuerza la idea del juego como libertad limitada por el sistema de reglas y mecánicas. El paralelismo existente con la premisa de Matrix y los interfaces que emplea potencian también esta interpretación metadiscursiva del videojuego. No obstante, como hemos estudiado a propósito de otros juegos (Rodríguez Serrano, Martín-Núñez y Gil-Soldevila, 2017), es probable pensar que el interés en el juego no descansa principalmente

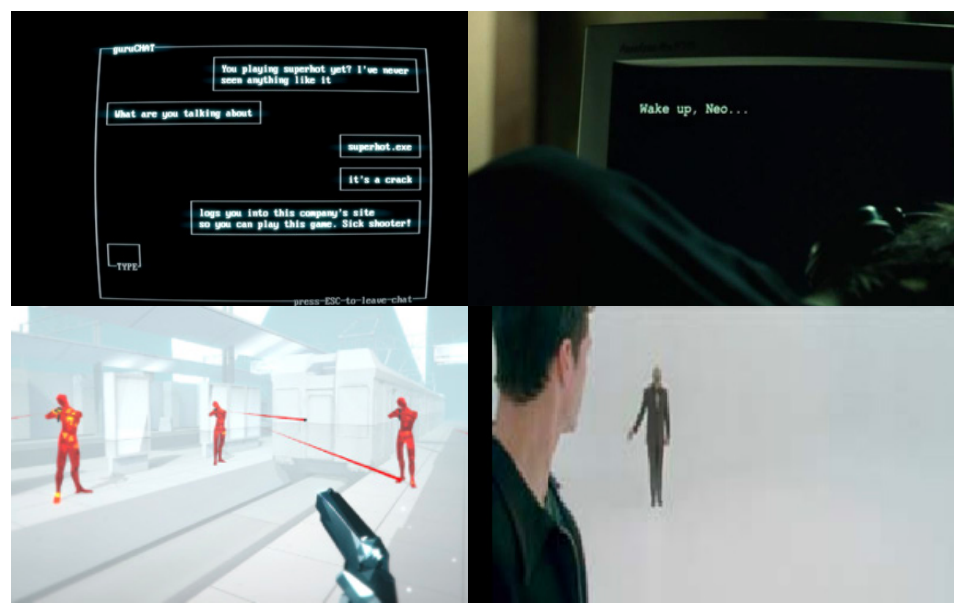

Fig. 3. A la izquierda los interfaces del sistema y simulación de SuperHOT (2016), SUPERHOT Team C), a la derecha, de Matrix (Hermanas Wachowski, 1999), Warner Bros. Entertainment Inc. (O). 
en la actualización narrativa de un universo conocido - el usuario contra el sistema - sino en la novedad mecánica que el juego propone.

\subsection{La reconfiguración espaciotemporal en SuperHOT VR}

El dispositivo tecnológico que media en el proceso de juego es una parte vital de este. Es condicionado y condicionante con respecto al resto del sistema y su funcionamiento. Por ello, nos interesa analizar también la adaptación a realidad virtual que se produce en SuperHOT VR y cómo reconfigura el sistema de juego en general y el gameworld en particular.

El cambio de plataforma implica que la mirada del jugador y la mirada de la cámara — que en este caso es además la mirada del avatar del jugador - se unifican en una entidad individual: la cámara ya no es controlada por el jugador a través de un dispositivo de entrada externo, la cámara es ahora la mirada del jugador. La consecuencia inmediata de esto es la imposibilidad de avanzar fácilmente por el espacio virtual, dado que el jugador no puede caminar fuera de los límites de la habitación en la que está. Es por esto que la configuración del espacio virtual cambia; ahora el jugador se encuentra limitado a ciertas zonas y desde allí combatirá y esquivará las balas que se dirigen al cuerpo de su avatar — ahora en perfecta sincronía con la localización física del jugador-.

La función que antes cumplía el movimiento del avatar alrededor del mapa para situarse en lugares protegidos se ve ahora sustituida por la necesidad de controlar todo aquello presente en el fuera de campo para proteger el cuerpo del avatar, que ahora es uno con el propio cuerpo. Es este grado de identificación cuerpo-avatar el que reformula el funcionamiento de la experiencia temporal en el juego a través de los dispositivos de realidad virtual. Ya no es necesario el movimiento para hacer avanzar el tiempo. El tiempo ahora avanza porque el jugador está entretejido con su propio avatar y, por tanto, el tiempo del mundo del jugador es ahora el del mundo virtual.

Como ocurre en el primer SuperHOT, el dispositivo que media entre el jugador y el juego se ve representado en el propio videojuego, pero ya no es a través del menú de un sistema operativo obsoleto, sino que en SuperHOT VR el avatar del jugador - en adelante, avatar de primer nivel - se coloca unas gafas de realidad virtual dentro de la propia diégesis del mundo virtual. El jugador se ve identificado con él al colocarse las gafas y sus tiempos de juego se sincronizan. Sin embargo, el tiempo del mundo virtual en el que está el avatar del avatar de primer nivel —en adelante avatar de segundo nivel—, o la diégesis dentro de la diégesis, sí se ve alterado con el movimiento del jugador. De esta forma el jugador ve su tiempo y el tiempo del avatar de primer nivel como una única entidad y controla con su movimiento el tiempo del mundo virtual, en el que se encuentra el avatar de segundo nivel.

Así, ya no es necesario que el tiempo siga pasando, aunque lentamente, cuando el jugador está detenido para mantener la fugacidad del tiempo de juego y la lógica de la imposibilidad de detener la partida en un instante. En SuperHOT V $\mathrm{R}$ las entidades del juego sí se paran completamente cuando el jugador se detiene. Porque parar el movimiento frena el movimiento del mundo virtual del avatar de segundo nivel, pero el avatar de primer nivel habita el mismo tiempo que el jugador. El tiempo no se para, solo se detiene el juego al que el avatar de primer nivel está jugando dentro de la propia diégesis. 


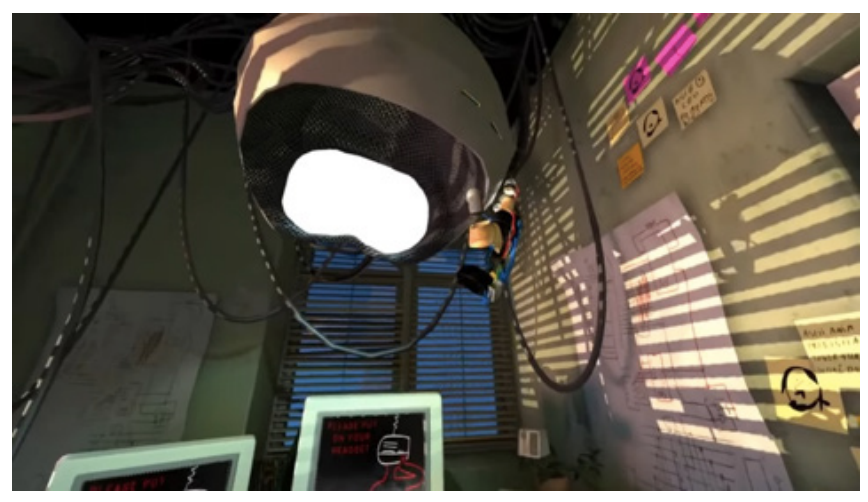

Fig. 4. Fotograma de SuperHOT VR (2018), SUPERHOT Team C), el avatar de primer nivel se coloca las gafas de realidad virtual.

Sin embargo, la nueva forma de interacción con la cámara refuerza la imposibilidad de parar el tiempo. Para controlar la mirada de la cámara-avatar, a diferencia de cuando se juega SuperHOT en una pantalla, el jugador necesita moverse. Ya no es posible rotar la mirada sin generar movimiento, porque esa mirada está anclada a un cuerpo físico y, al moverse el jugador, necesariamente, mueve su avatar en ambos niveles de abstracción virtual y, por tanto, el tiempo continúa. Ya no hay de posibilidad de pararse a pensar mientras se mira alrededor, porque el acto de mirar implica ahora movimiento, y el movimiento, tiempo.

Es así como el jugador da el paso de jugar el shooter como una partida de ajedrez a experimentar el interior de una partida de ajedrez en forma de shooter. El jugador ya no está en control de las piezas, el jugador ahora es la pieza de ajedrez que debe proteger su propia vida de la muerte para salir de un bucle en el que ya no se le pregunta si quiere "pulsar R" para reiniciar instantáneamente. En el momento de su muerte, una luz blanca lo cubre todo y sitúa al jugador de nuevo al inicio el nivel, construyendo aún más sobre la fugacidad del momento de la muerte en oposición a la repetición en forma de killcam del shooter tradicional.

\section{Discusión y conclusiones}

Como ha quedado patente en los resultados del análisis del caso de estudio, existe en los videojuegos una estrecha relación entre las estructuras espaciales y las temporales. SuperHOT va un paso más lejos, dando la apariencia de situar esta relación espaciotemporal en manos de la agencia del jugador, explicitándola así. Es en la dualidad de temporalidades que esto genera, junto con el aspecto visual del videojuego, donde empieza a construirse la idea de fugacidad que nos ocupa. Una fugacidad que, aunque puede parecer contradictoria con los bucles temporales del videojuego, no lo es, pues estos funcionan como catalizador del cambio en el comportamiento del jugador.

Igualmente importante es analizar la suerte de suspensión del tiempo por medio del movimiento que subvierte el planteamiento mecánico y el tempo del shooter tradicional en SuperHOT. El gameworld parece quedar a merced del jugador, pero este control es solo una ilusión falaz que se ve limitada por las reglas del mundo virtual que, en realidad, no otorgan el control total del vector espaciotemporal al jugador. Aquí, al combinar esta idea con la ausencia de menú de pausa y con el discurso propuesto por la narrativa estructurada de juego, surge una lectura metadiscursiva de SuperHOT que explora los límites de la libertad en los mundos virtuales. 
Por último, cabe plantear que el paso a los dispositivos de realidad virtual reconfigura necesariamente el sistema central del videojuego y, en consecuencia, también el mundo virtual en el que se sitúan el espacio y el tiempo. Esto ha quedado suficientemente probado para el objeto de análisis, pero esta reconfiguración del sistema central con respecto del videojuego tradicional es un proceso característico de la realidad virtual cuyo estudio en otros videojuegos podría aportar más resultados en este sentido. Impulsando la avataridad del jugador mediante estos dispositivos, se vincula el tiempo del juego con el tiempo del jugador, generando textos videolúdicos cuya lectura espaciotemporal es diferente a la de los videojuegos que utilizan una pantalla como dispositivo tecnológico de salida. Así se ve modificada y reformulada la relación entre el tiempo de lectura y el tiempo de la propia diégesis, y en SuperHOT V R el jugador pasa a vivir desde el interior de una ficha el juego de tablero fugaz que es, en definitiva, SuperHOT.

\section{Bibliografía}

Bushnell, R. (2016). Tragic Time in Drama, Film, and Videogames. The future in the Instant. London: Palgrave McMillan.

Cabañes, E. (2013). La percepción del espacio en los videojuegos: transgrediendo los límites de la pantalla. Bity aparte. Revista interdisciplinar de estudios videolúdicos, 0. 64-75.

Dubois, P. (2015). El acto fotográfico y otros ensayos. Buenos Aires: La marca editora.

Elsaesser, T. (2009). The Mind-Game Film. En W. Buckland (ed.) Puzzle Films. Complex Storytelling In Contemporary Cinema. West Sussex: Wiley-Blackwell.

Elsaesser, T. (2013). Los actos tienen consecuencias. Lógicas del mindgame films en la trilogía de Los Ángeles de David Lynch. L'Atalante, revista de estudios cinematográficos, 15, 7-18.

Eskelinen, M. (2012): Cybertext poetics. The Critical Landscape of New Media Literary Theory. London \& New York: The Continuum International Publishing Group.

Fernández-Vara, C. (2015). Introduction to Game Analysis. New York: Routledge.

Gazzard, A. (2011). Unlocking the Gameworld: The Rewards of Space and Time in Videogames. Game Studies. The international journal of computer game research, 11(1).

Genette, G. (1980). Narrative Discourse. An Essay in Method. Ithaca, New York: Cornell University Press.

Gómez-Tarín, F. J. (2011). Elementos de narrativa audiovisual: expresión y narración. Santander: Shangrila Ediciones.

Hanson, C. (2018). Game Time. Understanding Temporality in Video Games. Indiana: Indiana University Press.

Jenkins, H. (2004). Game Design as Narrative Architecture. En: N. Wardrip-Fruin and P. Harrigan (eds.) New Media as Story, Performance and Game, pp.118-130. Cambridge \& London: MIT Press. 
Juul, J. (2013). The Art of Failure. Cambridge \& London: MIT Press.

Lindley, C. (2005). The Semiotics of Time Structure in Ludic Space As a Foundation for Analysis and Design. Game Studies. The international journal of computer game research, 5(1).

Martín Núñez, M.; García Catalán, S.; Rodríguez Serrano A. (2016). Sí, la vida es muy rara. La culpa y el tiempo en Life is Strange. Analisi: Quaderns de Comunicació i Cultura, 54. DOI: http:// dx.doi.org/10.7238/a.v0i54.2908

Monchán, J. (2014). Juégame un cuento. En: VV.AA. Del tilt al byte. Videojuegos. Valencia: Diputación de Valencia, 79-82.

Navarro-Remesal, V. (2015). ¿La vida empieza o acaba con el matrimonio?: Amor, diseño de ética y libertad dirigida en Catherine. adComunica. Revista Cientifica de Estrategias, Tendencias e Innovación en Comunicación, 9, pp. 43-61. DOI: http://dx.doi.org/10.6035/2174-0992.2014.9.4.

Navarro-Remesal, V. (2016). Libertad dirigida. Una gramática del análisis y diseño del videojuego. Santander: Shangrila.

Nitsche, M. (2007). Mapping Time in Video Games. Situated Play, Proceedings of DiGRA 2007 Conference, 4.

Nitsche, M. (2008). Video Game Spaces. Image, Play, and Structure in $3 D$ Game Worlds. Cambridge \& London: The MIT Press.

Planells, A. J. (2015). Videojuegos y mundos de ficción. De Super Mario a Portal. Madrid: Cátedra.

Rodríguez Serrano, A.; Martín-Núñez, M.; Gil-Soldevila, S. (2017). Diseño ludológico y realidad aumentada. La experiencia de juego en Pokémon Go! (Niantic, 2016). Revista Latina de Comunicación Social, 72, 667-678. DOI: http://dx.doi.org/10.4185/RLCS-2017-1185

Ruiz Collantes, X. (2008). Juegos y videojuegos: formas de vivencias narrativas. En C. Scolari (ed). L'bomo videoludens: videojocs, textualitat i narrativa interactiva. Vic: Eumo, 15-52.

Sorolla-Romero, T. (2018). Narrativas no lineales. Entre la reconstrucción del MRI fracturado y la evidencia de su artificialidad. Tesis doctoral. Castellón: Universitat Jaume I.

Sora, C. (2016). Temporalidades digitales: análisis del tiempo en los new media y las narrativas interactivas. Barcelona: UOC.

Vargas-Iglesias, J.J. (2019). Semiotic and discursive consequences of the cybertextual condition: The case of tragedy. Semiotica. Journal of the International Association for Semiotic Studies, 229, 329352. https://doi.org/10.1515/sem-2017-0114

\section{Ludografía y filmografía}

Barr, P. (2011). Let's Play: Ancient Greek Punishment [Videojuego].

Berman, B. (productor) y hermanas Wachowski (directoras) (1999). The Matrix [cinta 
cinematográfica]. EE.UU.: Warner Brothers.

Blow, J. (2008). Braid [Videojuego]. Estados Unidos: Number None.

Cage, D. (Director) (2013). Beyond: Two Souls [Videojuego]. Francia: Quantic Dream.

Cyclone Studios (Desarrolladora) (1999). Requiem: Avenging Angel [Videojuego]. Francia: Ubisoft.

Dontnod Entertainment (Desarrolladora) (2015). Life is Strange [Videojuego]. Japón: Square Enix.

Fullbright (Desarrolladora) (2013). Gone Home [Videojuego]. Estados Unidos.

Kojima, H. (2019). Death Stranding [Videojuego]. Japón: Kojima Productions.

Pázhitnov, A. (1984). Tetris [Videojuego]. Unión Soviética.

Remedy Entertainment (Desarrolladora) (2001). Max Payne [Videojuego]. Estados Unidos: Gathering of Developers.

Skorupka, C. (Guionista) Iwanicki, Piotr; Rriska, Panos (Diseñadores) (2016). SuperHOT [Videojuego]. Polonia: SUPERHOT Team.

Skorupka, C. (Guionista) Iwanicki, Piotr; Rriska, Panos (Diseñadores) (2018). SuperHOT VR [Videojuego]. Polonia: SUOERHOT Team.

Wolpaw, E.; Faliszek, C. (Guionistas) Swift, Kim (Diseñadora) (2007). Portal [Videojuego]. Estados Unidos: Valve.

Wreden, D.(2011). The Stanley Parable [Videojuego]. Estados Unidos: Galactic Café. 


\section{Uso de imágenes}

Las imágenes utilizadas como figuras en este artículo son elementos centrales del análisis, realizado únicamente con fines científicos en el ámbito académico.

\section{Fuentes de financiación}

El presente trabajo ha sido realizado en el marco de los proyectos de investigación $\mathrm{E} /$ diseño narratológico en videojuegos: una propuesta de estructuras, estilos y elementos de creación narrativa de influencia postclásica (DiNaVi) (código 18I369.01/1), dirigido por Marta Martín-Núñez y Análisis de identidades discursivas en la era de la posverdad. Generación de contenidos audiovisuales para una Educomunicación crítica (AIDEP) (código 18I390.01/1) dirigido por Javier Marzal Felici. Ambos fueron financiados por la Universitat Jaume I, a través de la convocatoria competitiva de proyectos de investigación de la UJI, para el periodo 2019-2021.

Este trabajo ha sido realizado en el marco del programa Estudia e Investiga de la Universitat Jaume I. 
MHCJ Vol. 11 (2) | Año 2020 - Artículo n¹1 (162) - Páginas 221 a 238 - mhjournal.org

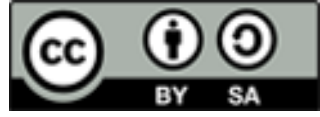

Licencia Creative Commons

Miguel Hernández Communication Journal

mhjournal.org

\section{Cómo citar este texto:}

Diego Villabrille Seca y Marta Martín Nuñez (2020): Lo fugaz del instante jugable en los mundos virtuales. Espacialidad y temporalidad en SuperHOT y SuperHOT VR, en Miguel Hernández Communication Journal, Vol. 11 (2), pp. 221 a 238. Universidad Miguel Hernández, UMH (Elche-Alicante). DOI: http:// dx.doi. org/10.21134/mhcj.v11i0.340 